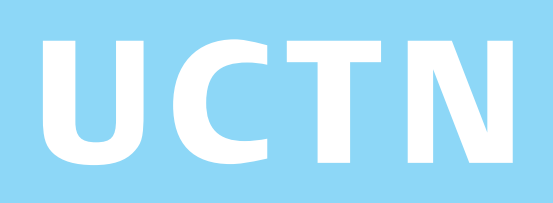

\title{
Esophageal duplication cyst presenting as a submucosal tumor: diagnosis by endoscopic ultrasound-guided fine-needle aspiration
}

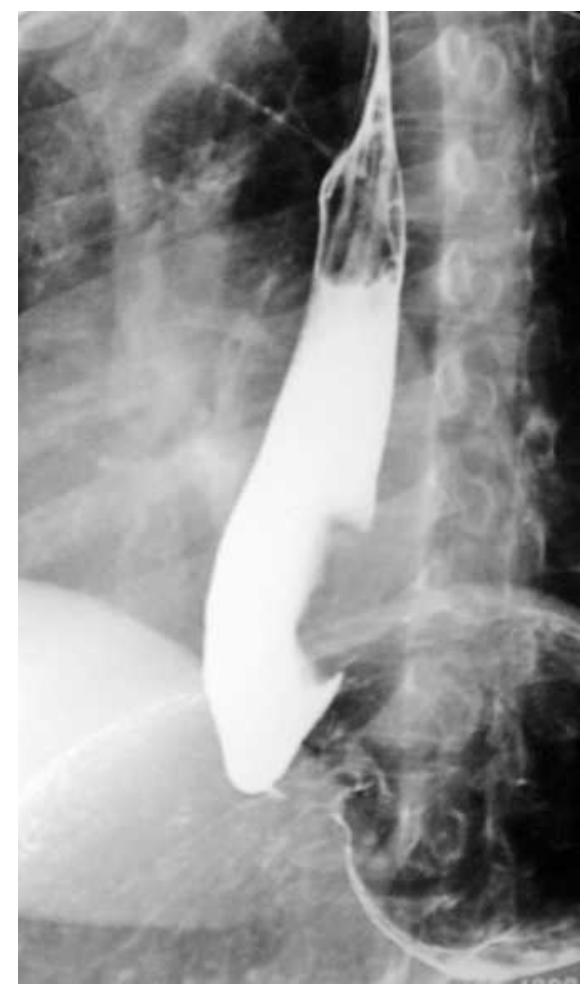

Figure 1 An upper gastrointestinal series revealed a smooth, wide-based indentation of the outline at the level of the lower esophagus.

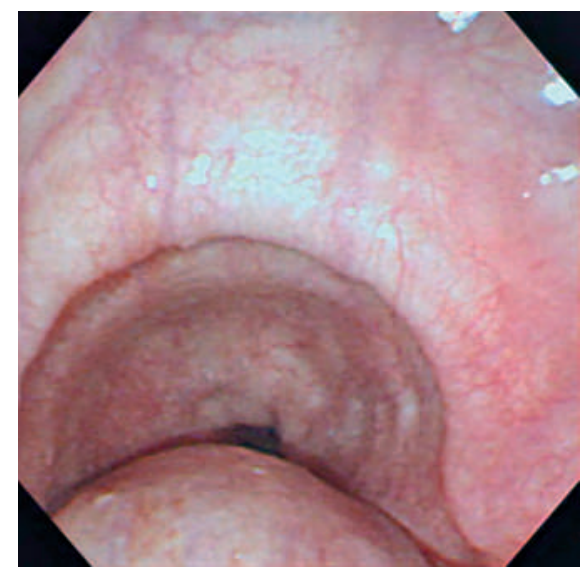

Figure 2 Esophagogastroduodenoscopic view showed a submucosal tumor with normal-looking overlying mucosa in the lower esophagus.

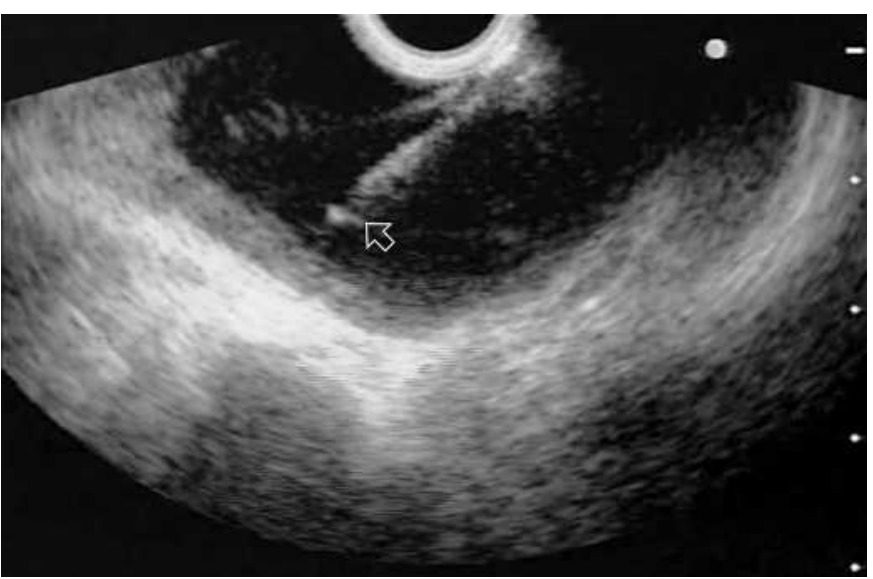

Figure 3 The endoscopic ultrasound (EUS) image revealed a $3.4-\mathrm{cm}$ anechoic lesion with a thick wall in the third layer of lower esophagus. EUSguided fine-needle aspiration (EUSFNA) (arrow) yielded a large volume of dark-yellowish mucoid material.

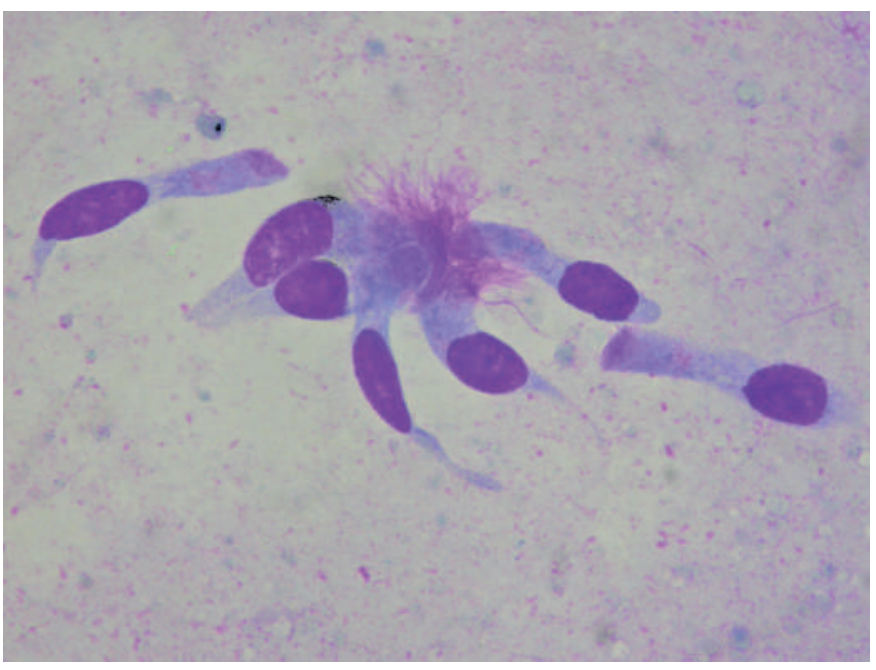

Figure 4 EUS-FNA cytology showed groups of ciliated columnar cells against a mucoid, cystic background (Hemacolor stain, original magnification $\times 400$ ).

A 44-year-old woman presented with symptoms of intermittent epigastric pain and chest discomfort which she had suffered for more than 5 years. She had noticed progressive discomfort on swallowing in the past 1 month. The episodic attacks were not related to meals, respiration, or exercise. She underwent esophagogastroduodenoscopy at her local hospital and this revealed a suspicious-looking region of external compression over the lower esophagus. Chest computed tomography showed a $3.4-\mathrm{cm}$ mediastinal tumor that was adhering to the lower esophagus. She was then referred to our hospital for further evaluation.

An upper gastrointestinal series showed a smooth, wide-based indentation of the outline at the level of the lower esopha- gus (Figure 1), and repeat esophagogastroduodenoscopy showed a submucosal tumor with normal-looking overlying mucosa (Figure 2). Endoscopic ultrasound (EUS) was performed with an Olympus UC2000P echo endoscope (Olympus, Tokyo, Japan) in an attempt to clarify the character of the submucosal tumor. The EUS image revealed a $3.4-\mathrm{cm}$, anechoic lesion with a thick wall in the third layer of the lower esophagus. Subsequent EUSguided fine-needle aspiration using a 22gauge Olympus MAJ-919 needle yielded a large volume of dark-yellowish mucoid material (Figure 3). On-site cytology after staining with Hemacolor stain (Merck, Darmstadt, Germany) showed groups of

DOI: $10.1055 / s-2006-944865$ 
ciliated columnar cells on a mucoid cystic background (Figure 4). There were no lymphocytes or polymorphonuclear leukocytes. These findings were consistent with a diagnosis of esophageal duplication cyst. The patient eventually underwent a surgical resection procedure for symptomatic relief, and the histopathologic examination of the resection specimen confirmed the diagnosis.

This case demonstrates that EUS with fine-needle aspiration can provide a quick, accurate, and minimally invasive method for the diagnosis of esophageal duplication cyst.

Endoscopy_UCTN_Code_CCL_1AF_2AB

Endoscopy_UCTN_Code_CCL_1AB_2AC_3AH
T.-Y. Cheng', ${ }^{1,2}$ J.-H. Chen ${ }^{3}$, I.-S. Jan', C.-C. Chen ${ }^{2}$, H.-P. Wang ${ }^{4}$, J.-T. Lin ${ }^{2}$

${ }^{1}$ Department of Laboratory Medicine, National Taiwan University Hospital and National Taiwan University College of Medicine, Taipei, Taiwan

2 Department of Internal Medicine, National Taiwan University Hospital and National Taiwan University College of Medicine, Taipei, Taiwan

${ }^{3}$ Department of Internal Medicine, TzuChi General Hospital, Taipei Branch, Taiwan

${ }^{4}$ Department of Emergency Medicine, National Taiwan University Hospital and National Taiwan University College of Medicine, Taipei, Taiwan.
Corresponding Author

\section{H.-P. Wang, M.D.}

Department of Emergency Medicine National Taiwan University Hospital 7 Chung-Shan South Road

Taipei

Taiwan

Fax: $\quad$ +886-2-23947899

E-mail: whp@ha.mc.ntu.edu.tw 